\title{
Safety Profile of Ombitasvir/Paritaprevir/Ritonavir and Dasabuvir in Hepatitis C in Romania: an Analysis Conducted in VigiBase ${ }^{\circledast}$ WHO
}

\author{
Irina Cazacu ${ }^{1}$, Roxana Stroe ${ }^{2}$, Roxana Dondera $^{2}$, Bogdan Apan $^{3}$, Andreea Farcas $^{4}$, Anamaria Cristina $^{1}$, Camelia Bucsa $^{4}$, \\ Cristina Mogosan ${ }^{1,4}$
}

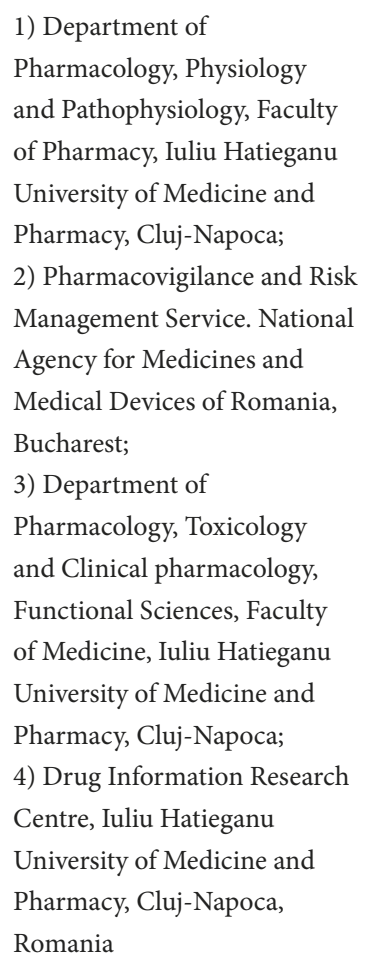

1) Department of

Pharmacology, Physiology and Pathophysiology, Faculty of Pharmacy, Iuliu Hatieganu University of Medicine and Pharmacy, Cluj-Napoca;

2) Pharmacovigilance and Risk Management Service. National Agency for Medicines and Medical Devices of Romania, Bucharest;

3) Department of Pharmacology, Toxicology and Clinical pharmacology, Functional Sciences, Faculty of Medicine, Iuliu Hatieganu University of Medicine and Pharmacy, Cluj-Napoca;

4) Drug Information Research Centre, Iuliu Hatieganu University of Medicine and Pharmacy, Cluj-Napoca, Romania

\footnotetext{
Address for correspondence: Irina Cazacu

Department of Pharmacology, Physiology and

Pathophysiology, Faculty of Pharmacy, Iuliu Hatieganu University of Medicine and Pharmacy, 6A Pasteur Street, Cluj-Napoca, Cluj County, Romania cazacuirina16@gmail.com
}

Accepted: 02.10.2020

\section{ABSTRACT}

Background \& Aims: Due to the increasing number of hepatitis C virus (HCV)-infected patients being treated with direct antiviral agents (DAAs) in Romania, we aimed to conduct a pharmacovigilance study in order to comprehensively evaluate the safety profile for the ombitasvir/paritaprevir/ritonavir and dasabuvir $(\mathrm{Om} / \mathrm{Pa} / \mathrm{Ri}+\mathrm{Da})$ regimen.

Methods: A retrospective analysis was conducted on the individual case safety reports (ICSRs), extracted from VigiBase ${ }^{\infty}$ on $1^{\text {st }}$ February 2018, which included the $\mathrm{Om} / \mathrm{Pa} / \mathrm{Ri}+\mathrm{Da}$ regimen as suspected for causing adverse drug reactions (ADRs). Potential drug-drug interactions (DDIs) were checked for all concomitant medication using the IBM Micromedex tool.

Results: Among the 1,102 ICSRs retrieved, 260 were serious (23.5\%). Ribavirin was significantly associated with more ADRs reported per case (on average 5.1 vs. 3.2 ADRs/case, $\mathrm{p}<0.001$ ). Most commonly reported ADRs were pruritus (6.8\%), fatigue (5.4\%), dizziness (4.3\%) and headache (3.8\%). For the serious ICSRs, a significant relationship was found between age and renal and urinary disorders, and between gender and neoplasms, injury, poisonings and procedural complications. Hepatotoxicity was identified in six ICSRs, four of them being serious. Potential contraindicated DDIs were identified in 1.9\% of all ICSRs and major DDIs in 20.4\%. Conclusions: About a third of the ICSRs related to $\mathrm{Om} / \mathrm{Pa} / \mathrm{Ri}+\mathrm{Da}$ regimen were serious. Pruritus, fatigue, dizziness and headache were the most commonly reported ADRs. The frequent use of multiple comedications in HCV-infected patients requires the consideration of potential DDIs when using the Om/ $\mathrm{Pa} / \mathrm{Ri}+\mathrm{Da}$ regimen.

Key words: hepatitis C - direct antiviral agents - Romania - drug-related side effects - adverse reactions drug interactions - ombitasvir/paritaprevir/ritonavir - dasabuvir

Abbreviations: ADRs: adverse drug reactions; AEs: adverse events; CNS: central nervous system; DAAs: direct antiviral agents; DDIs: drug-drug interactions; HBV: hepatitis B virus; HCC: hepatocellular carcinoma; HCV: hepatitis C virus; HLT: high level term; ICSRs: individual case safety reports; MedDRA: Medical Dictionary for Regulatory Activities; NEC: not elsewhere classified; NHIH: National Health Insurance House; Om/Pa/ $\mathrm{Ri}+\mathrm{Da}$ : ombitasvir/paritaprevir/ritonavir and dasabuvir; PT: preferred term; SOC: system organ class; UMC: Uppsala Monitoring Centre; WHO: World Health Organization.

\section{INTRODUCTION}

Hepatitis C virus (HCV) infection affects $4.5-4.9 \%$ of the general population in Romania, comprising the highest viraemic prevalence in Europe, and has been identified as the main cause of chronic hepatitis (64\%), liver cirrhosis (59\%) and liver transplantation $(31.5 \%)$ in Romanian patients [1-3].
Direct antiviral agents (DAAs) represent a major clinical advance, by achieving sustained virological response or cure in more than $95 \%$ of the HCV-infected patients. Direct antiviral agents have proved effective and generally well tolerated by patients, showing a reduction in the rates of liver complications, hepatocellular carcinoma (HCC), and liver-related mortality [1]. In addition, DAAs also demonstrated benefits in reducing morbidity caused by renal, autoimmune, and metabolic complications [3]. However, adverse drug reactions (ADRs) are known to occur in more than $77 \%$ of patients treated with DAAs, and sometimes serious ADRs have been associated with poor treatment compliance and with treatment discontinuation 
$[4,5]$. Drug-drug interactions (DDIs) are of particular concern in $\mathrm{HCV}$-infected patients, because they may increase the frequency and seriousness of ADRs [6, 7].

The DAAs treatment for HCV genotype 1 patients with Child Pugh class A cirrhosis has been available in Romania since December 2015, based on cost-volume-outcome contracts concluded by National Health Insurance House (NHIH). Hepatitis $\mathrm{C}$ virus genotype $1 \mathrm{~b}$ was found to be almost exclusively present (99.6\%) in Romanian patients with HCV and advanced fibrosis, eligible for DAAs treatment [1]. The number of patients who have benefited from these treatments through NHIH has constantly increased, from 5,800 patients being treated in 2015-2016 to 12,000 patients in 2016-2017 [8]. Ombitasvir/paritaprevir/ritonavir and dasabuvir (Om/ $\mathrm{Pa} / \mathrm{Ri}+\mathrm{Da}$ ), with or without ribavirin, was the only DAAs treatment regimen available in Romania until May 2017; later other DAAs were introduced.

Due to the increasing number of patients being treated with DAAs, a pharmacovigilance study to outline the safety profile of these medicines for the Romanian patients was required. Therefore, the present study aimed to comprehensively evaluate the safety profile for the $\mathrm{Om} / \mathrm{Pa} / \mathrm{Ri}+\mathrm{Da}$ regimen, with or without ribavirin, in hepatitis $\mathrm{C}$ Romanian patients, with a focus on gender, age, serious ADRs and on the important potential and identified risks.

\section{METHODS}

A descriptive, retrospective analysis was conducted on the individual case safety reports (ICSRs) registered in the VigiBase ${ }^{\varpi}$, the World Health Organization (WHO) international database. VigiBase includes the suspected ADRs received from national pharmacovigilance centers around the world, being developed and maintained by the Uppsala Monitoring Centre (UMC), the WHO Collaborating Centre for International Drug Monitoring [9]. Around 17 million ICSRs from 130 countries had been compiled in VigiBase up to 2018 [10]. The information in VigiBase comes from a variety of sources, and the probability that the suspected adverse event is drug-related is not the same in all cases. The study results and conclusions are those of the authors and not necessarily those of the UMC or the WHO.

An extraction of VigiBase data was performed on $1^{\text {st }}$ February 2018, which included all ICSRs from Romania for the association $\mathrm{Om} / \mathrm{Pa} / \mathrm{Ri}+\mathrm{Da}$, suspected for causing ADRs. The following data was included in the analysis: reporter, patients' age and gender, ADRs described according to the Medical Dictionary for Regulatory Activities (MedDRA terminology 21.0) by System Organ Class (SOC), High Level Term (HLT) and Preferred Term (PT), outcome for each ADR, seriousness, seriousness criteria, co-reported medicines (coded according to the WHODrug ${ }^{\mathrm{Tm}}$ dictionary) and duration of treatment. Medicines were listed as being "suspected" (drugs suspected for the reaction, but not explicitly due to a drug interaction), "interacting" (if an ADR is suspected of being related to a drug interaction between two or more drugs) or "concomitant" (drugs used concurrently but not suspected by the reporter to have caused the ADR) [11]. A case could contain more than one
$\mathrm{ADR}$ and more than one seriousness criterion. An ADR was considered serious if it resulted in death, was life-threatening, required hospitalization or prolonged hospitalization, involved persistent or significant disability or incapacity, caused congenital anomaly or birth defects, or was considered medically significant by the reporter [12].

Potential DDIs were checked for all concomitant medication using the "Drug Interactions" tool in IBM Micromedex ${ }^{\oplus}$ [13]. Only major and contraindicated potential DDIs were further analyzed for seriousness and outcome. A DDI considered "contraindicated" means that "the drugs are contraindicated for concurrent use", while a "major" DDI means that "the interaction may be life-threatening and/or require medical intervention to minimize or prevent serious adverse effects" [12].

Descriptive statistics were used to characterize the variables assessed in this study. Qualitative variables were presented by counts and percentages and continuous variables by median and range. The frequencies of ADRs by SOC, HLT and PT were compared between genders, and age groups, $<70$ years old and $\geq 70$ years old, using Pearson 's chi-squared test. When the $\mathrm{p}$ value was $<0.05$, differences were considered statistically significant.

\section{RESULTS}

A total of 1,102 ICSRs were retrieved from VigiBase and among them, 260 were serious (23.5\%). The main reporters, mostly through facilitated collection, were the consumers (80.6\%). Patients had a 64 years median age (range 24-88, unknown in $32.5 \%$ of ICSRs) and there were more women $(\mathrm{n}=761,69 \%)$; the gender was unknown in 27 ICSRs $(2.5 \%)$ (Table I). We identified 258 "concomitant" medicines in 909 ICSRs, which included mainly ribavirin (49.4\%), metoprolol (12.3\%), candesartan (7.8\%) and indapamide (7.4\%). Ribavirin was "suspected" in 131 ICSRs. Five ICSRs had other "suspected" medications, aside $\mathrm{Om} / \mathrm{Pa} / \mathrm{Ri}+\mathrm{Da}$ and ribavirin. Only one ICSR had a drug coded as "interacting" (pentoxifylline).

The 1,102 ICSRs retrieved from VigiBase described in total 4,608 ADRs, with an average of 4.1 ADRs per case (range: 1-36). The presence of ribavirin as concomitant medication was significantly associated with more ADRs reported (on average 5.1 vs. $3.2 \mathrm{ADRs} /$ case, $\mathrm{p}<0.001$ ). Among the ADRs reported, the highest percentage belonged to gastrointestinal disorders (18.2\%), general disorders and administration site conditions (14.6\%), nervous system disorders (14.1\%) and skin and subcutaneous tissue disorders (10.4\%) (Table I). The most frequent ADRs according to HLT were asthenic conditions (9.2\%), pruritus not elsewhere classified (NEC) (6.9\%), nausea and vomiting symptoms (6.5\%) and neurological signs and symptoms NEC (3.8\%). Most commonly reported PTs were pruritus (6.8\%), fatigue (5.4\%), dizziness $(4.3 \%)$ and headache $(3.8 \%)$. The outcome at the time of reporting of ADRs was mainly not recovered (52.9\%), followed by recovered (20.0\%), recovering (16.3\%), death $(2.1 \%)$, recovered with sequelae $(0.1 \%)$; unknown outcome was reported in $8.3 \%$.

By SOCs, the gastrointestinal disorders, $19.1 \%$ in female and $16.2 \%$ in male, were followed by nervous system 
Table I. Characteristics of individual case safety reports according to gender and age category.

\begin{tabular}{|c|c|c|c|c|c|c|c|}
\hline Parameters & $\begin{array}{l}\text { Total } \\
\mathrm{n}(\%)\end{array}$ & $\begin{array}{c}\text { Female } \\
\mathrm{n}(\%)\end{array}$ & $\begin{array}{l}\text { Male } \\
\mathrm{n}(\%)\end{array}$ & & $\begin{array}{c}<70 \text { years old } \\
n(\%)\end{array}$ & $\begin{array}{c}\geq 70 \text { years old } \\
n(\%)\end{array}$ & \\
\hline Total number of ICSRs & $1,102(100)$ & $761(69.1)$ & $314(28.5)$ & & $546(49.5)$ & $198(18.0)$ & \\
\hline ICSRs including ribavirin & $545(49.4)$ & $342(62.8)$ & $184(33.8)$ & * & $343(62.9)$ & $119(21.8)$ & $* * *$ \\
\hline Serious ICSRs reported & $260(23.5)$ & $171(65.8)$ & $69(26.5)$ & $* * *$ & $123(47.3)$ & $71(27.3)$ & $* * *$ \\
\hline Caused/prolonged hospitalization & $212(19.2)$ & $148(69.8)$ & $61(28.8)$ & $* * *$ & $114(53.8)$ & $61(28.8)$ & \\
\hline Death & $33(2.9)$ & $23(69.7)$ & $10(30.3)$ & & $16(48.5)$ & $12(36.4)$ & \\
\hline Life threatening & $23(2.0)$ & $15(65.2)$ & $8(34.8)$ & & $15(65.2)$ & $6(26.1)$ & \\
\hline Disabling/incapacitating & $6(0.5)$ & $4(66.7)$ & $2(33.3)$ & & $6(100)$ & $0(0.0)$ & \\
\hline Other & $82(7.4)$ & $48(58.5)$ & $17(20.7)$ & $* * *$ & $33(40.2)$ & $13(15.9)$ & $* * *$ \\
\hline Total ADRs (\%) by SOC & $4,608(100)$ & $3,347(72.6)$ & $1,221(26.5)$ & & $2,561(55.6)$ & $984(21.4)$ & \\
\hline Gastrointestinal dis. & $841(18.2)$ & $640(76.1)$ & $198(23.5)$ & & $472(56.1)$ & $168(20.0)$ & \\
\hline General dis. and administration site cond. & $674(14.6)$ & $477(70.8)$ & $188(27.9)$ & & $360(53.4)$ & $154(22.8)$ & \\
\hline Nervous system dis. & $652(14.1)$ & $495(75.9)$ & $155(23.8)$ & & $379(58.1)$ & $127(19.5)$ & \\
\hline Skin and subcutaneous tissue dis. & $481(10.4)$ & $353(73.4)$ & $127(26.4)$ & & $238(49.5)$ & $88(18.3)$ & $* * *$ \\
\hline Investigations & $284(6.1)$ & $193(68.0)$ & $90(31.7)$ & & $151(53.2)$ & $57(20.1)$ & \\
\hline Psychiatric dis. & $281(6.0)$ & $185(65.8)$ & $94(33.5)$ & * & $172(61.2)$ & $55(19.6)$ & \\
\hline Musculoskeletal and connective tissue dis. & $246(5.3)$ & $176(71.5)$ & $69(28.0)$ & & $140(56.9)$ & $52(21.1)$ & \\
\hline Metabolism and nutrition dis. & $187(4.0)$ & $135(72.2)$ & $51(27.3)$ & & $106(56.7)$ & $43(23.0)$ & \\
\hline Respiratory, thoracic and mediastinal dis. & $179(3.8)$ & $138(77.1)$ & $41(22.9)$ & & $110(61.5)$ & $36(20.1)$ & \\
\hline Hepatobiliary dis. & $150(3.2)$ & $106(70.7)$ & $42(28.0)$ & & $89(59.3)$ & $41(27.3)$ & $* *$ \\
\hline
\end{tabular}

disorders (14.7\%) and general disorders and administration site conditions (14.2\%) in women, and general disorders and administration site conditions (15.3\%) and nervous system disorders $(12.6 \%)$ in men. Taking into consideration the age, by SOC, gastrointestinal disorders, in patients $<70$ years old, were followed by nervous system disorders (14.7\%), and general disorders and administration site conditions (14\%), and in patients $\geq 70$ years old by general disorders and administration site conditions (15.6\%) and nervous system disorders (12.9\%). There were more ADRs reported in patients aged $\geq 70$ years old, than in younger (on average 4.9 ADRs/ patient versus 4.6).

The 260 serious ICSRs described in total 579 ADRs, of which the most frequently reported ADRs belonged, by SOC to gastrointestinal disorders $(20.6 \%)$, followed by general disorders and administration site conditions (13.2\%), nervous system disorders (12.2\%) and hepatobiliary disorders (6.9\%). Among the 579 ADRs, the most frequently ADRs according to HLT were: asthenic disorders (7\%), nausea and vomiting symptoms (6.1\%), neurological signs and symptoms NEC (3.1\%) and appetite disorders (2.8\%). The most commonly reported ADRs, presented by $\mathrm{PT}$, were vomiting (3.2\%), dizziness $(3.1 \%)$, asthenia $(3.0 \%)$ and nausea $(2.8 \%)$. More serious ICSRs $(35.8 \%)$ and deaths $(6.0 \%)$ were reported in patients aged $\geq 70$ years old, than in patients aged $<70$ years old $(22.5 \% ; 2.9 \%)$.

Hepatotoxicity (liver damage) was identified in six ICSRs, four of which were serious; hepatocellular carcinoma was reported in six ICSRs, hepatic cancer in five and hepatic neoplasm in eight ICSRs. Hepatic failure was reported in 27 serious ICSRs (2.4\%), 12 of which were fatal (44.4\%). In five
ICSRs, co-infection with hepatitis B virus (HBV) was reported. There was no HBV reactivation reported.

As can be seen in Table I, there was a significant relationship between gender and psychiatric disorders, $\mathrm{X}^{2}(2)=6.53$, $p<0.0314$, between age and skin disorders, $X^{2}(2)=20.13$, $\mathrm{p}<0.001$, and between age and hepatobiliary disorders, $\mathrm{X}^{2}(2)=8.82, \mathrm{p}=0.0108$. The relation between serious ICSRs and age, $\mathrm{X}^{2}(2)=12.91, \mathrm{p}<0.001$, respectively gender, $\mathrm{X}^{2}(2)=17.37$, $\mathrm{p}<0.001$, was also significant. For the serious ICSRs, Fig. 1 shows that there was a significant association between age and renal and urinary disorders $\left[\mathrm{X}^{2}(2)=15.56, \mathrm{p}<0.001\right]$, between gender and neoplasms $\left[\mathrm{X}^{2}(2)=56.60, \mathrm{p}<0.001\right]$, and between gender and injury, poisoning and procedural complications $\left[\mathrm{X}^{2}(2)=39.16, \mathrm{p}<0.001\right]$.

A total of 49 serious ICSRs were coded with death and/ or with life threatening seriousness criteria. These patients had a median age of 66 years (range: 44-79, unknown for 6 ICSRs), 33 were female (67.3\%) and 16 were male $(32.7 \%)$. For these cases, 388 ADRs were identified, with an average of 7.9 ADRs reported per ICSR (range: 1-36). Presented by SOC, most reported were: gastrointestinal disorders (18.5\%), hepatobiliary disorders (14.1\%), general disorders and administration site conditions (12.8\%) and cardiac disorders (9\%). By HLT, most reported ADRs were: asthenic conditions (5.9\%), hepatic failure and associated disorders (4.8\%), non-site specific gastrointestinal haemorrhages $(4.3 \%)$ and cholestasis and jaundice (4.1\%), and by PT: hepatic failure (3.6\%), cardio-respiratory arrest $(2.8 \%)$, asthenia $(2.5 \%)$ and jaundice $(2.3 \%)$.

Potential contraindicated DDIs identified for $\mathrm{Om} / \mathrm{Pa} / \mathrm{Ri}+\mathrm{Da}$ are presented in Table II. They involved nine concomitant 


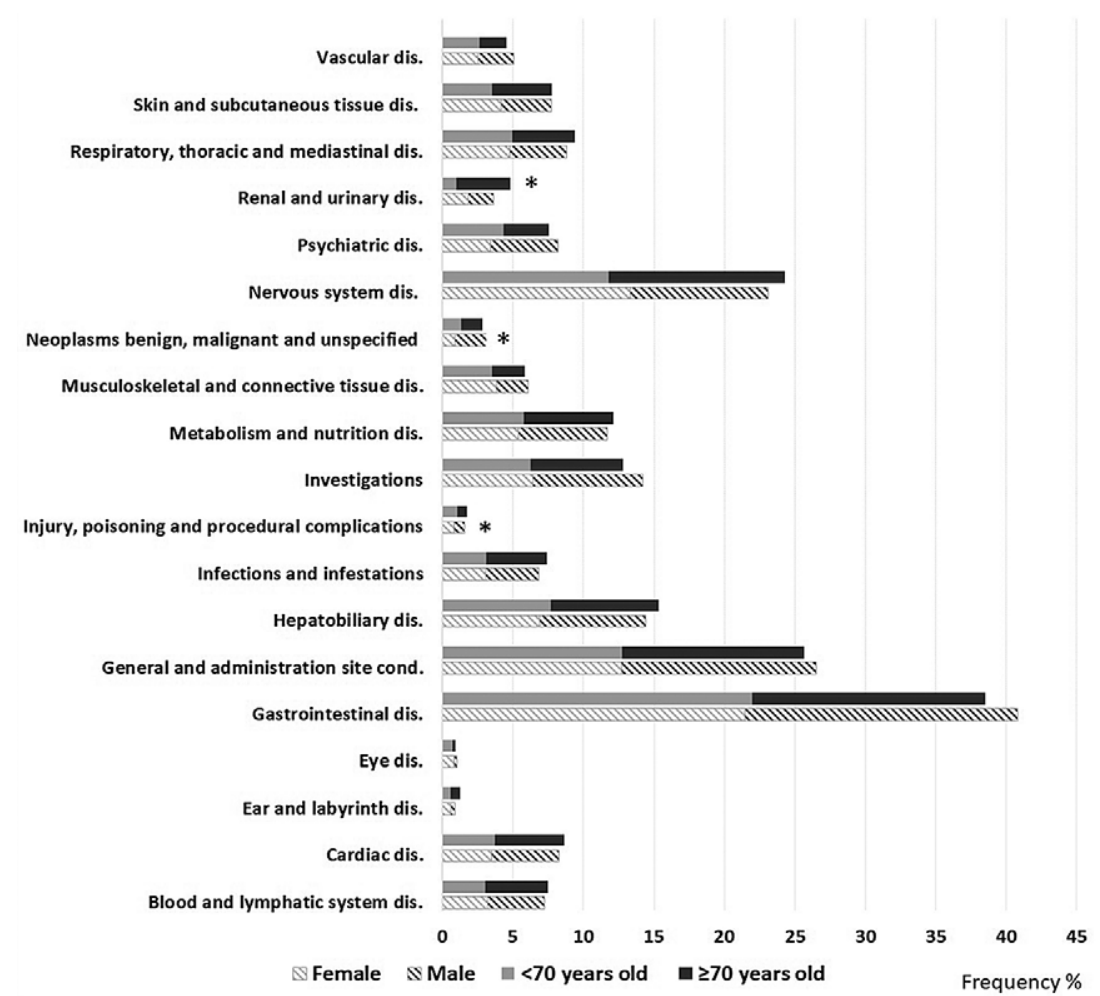

Fig. 1. Frequencies of serious ADRs, by System Organ Class (SOC), according to gender (female/male) and age category $(<70$ years old $/ \geq 70$ years old $) ;{ }^{*} \mathrm{p}<0.001$; dis. $=$ disorders.

medicines, which were identified in 21 ICSRs (1.9\%), 38\% of them being serious. For two concomitant medicines, flecainide and propafenone, we found potential outcomes related to the potential consequences of their interaction with ritonavir. Potential major DDIs (Supplementary material) were identified in 225 ICSRs (20.4\%), of which $36.8 \%$ were serious. Amlodipine $(\mathrm{n}=52)$ was the medicine most frequently involved in these latter ICSRs, followed by furosemide $(n=39)$, metformin $(n=25)$, omeprazole $(n=22)$ and digoxin $(n=13)$.

\section{DISCUSSION}

This is the first large pharmacovigilance analysis conducted on the safety of $\mathrm{Om} / \mathrm{Pa} / \mathrm{Ri}+\mathrm{Da}$ regimen in $\mathrm{HCV}$ infected Romanian patients, by using the WHO VigiBase. Previous clinical studies conducted in Romania focused on the efficacy and safety of $\mathrm{Om} / \mathrm{Pa} / \mathrm{Ri}+\mathrm{Da}$ regimen, by prospectively analyzing the first cohorts of patients during their first months of treatment, who started their therapy during December 2015 until July 2016; the samples varied from 59 to 2070 patients [14-17]. Analyzing the safety of $\mathrm{Om} / \mathrm{Pa} / \mathrm{Ri}+\mathrm{Da}$, these studies had some limitations: either they focused mainly on efficacy, included a limited number of patients, or they evaluated only the serious adverse events (AEs) that led to the discontinuation of antiviral therapy. Therefore, the present study offers an important insight into the safety profile of $\mathrm{Om} / \mathrm{Pa} / \mathrm{Ri}+\mathrm{Da}$ in Romanian treated patients, by including all ICSRs registered in WHO VigiBase until February 2018.

Different multicenter phase three clinical trials investigated the safety of $\mathrm{Om} / \mathrm{Pa} / \mathrm{Ri}+\mathrm{Da}$ and showed overall that the reported AEs were mild to moderate. Headache, nausea, fatigue, insomnia, diarrhea, asthenia and pruritus were the most common ADRs [18, 19]. Similarly, most commonly reported ADRs in our study were pruritus (6.8\%), fatigue (5.4\%), dizziness $(4.3 \%)$ and headache $(3.8 \%)$. These are usually symptoms commonly reported by patients in post-marketing pharmacovigilance system. Pruritus and headache were also among the most frequently reported ADRs in two prospective clinical studies conducted on Romanian patients treated with $\mathrm{Om} / \mathrm{Pa} / \mathrm{Ri}+\mathrm{Da}[14,15]$. However, Gheorghe et al. [16] reported anemia (33.5\%) as being the most frequently AE observed, followed by fatigue (18.7\%), pruritus (10.1\%), insomnia (8.4\%) and headache (5.4\%), while Trifan et al. [17] found that the most common AE was asthenia (20.4\%), followed by pruritus (10.5\%), insomnia (9.6\%) and headache (8.2\%). Another prospective clinical study conducted on 59 Romanian patients reported hyperbilirubinemia as the main $\mathrm{AE}$, observed in 33 out of the 59 patients included [12]. Increased bilirubin blood levels, anemia or asthenia were not among the most frequently observed ADRs in the ICSRs included in our study. The most frequently ADRs in our study could be explained by the high percentage of patients $(80.6 \%)$ from whom the ADR reports originated.

The study of Gheorghe et al. [16] showed significant higher percentage of AEs reported in women than in men $(42.9 \%$ vs $28.2 \%, p=0.0001)$. More ADRs were also reported for women $(69 \%)$ than for men $(28.5 \%)$ in our study. Statistical significance $(\mathrm{p}<0.05)$, in relation to the patients' gender, was found for psychiatric disorders for all ICSRs. Neoplasms benign, malignant and unspecified and injury, poisoning and procedural complications were significantly related to gender for the serious ICSRs. To our knowledge, this is the first study to evaluate in more detail the characteristics of ADRs related to the use of $\mathrm{Om} / \mathrm{Pa} / \mathrm{Ri}+\mathrm{Da}$ in women versus men. There could be 
Table II. Characteristics of potential drug-drug interactions, classified as contraindicated, and the seriousness of the individual case safety reports including them

\begin{tabular}{|c|c|c|c|c|c|}
\hline $\begin{array}{l}\text { Concomitant } \\
\text { reported medicines }\end{array}$ & $\begin{array}{l}\text { Interacting } \\
\text { medicines }\end{array}$ & Potential consequence of the interaction & $\mathrm{N}$ & $\begin{array}{l}\text { Seriousness }(\mathrm{N}) \\
\text { Type of seriousness } \\
\text { (N for each } \\
\text { criterion) }\end{array}$ & $\begin{array}{c}\text { Potential related } \\
\text { outcomes (PTs) }(\mathrm{N})\end{array}$ \\
\hline Amisulprid & Ritonavir & Increased risk of torsades de pointes & 2 & No & - \\
\hline Atorvastatin & Ritonavir & $\begin{array}{l}\text { Increased risk of myopathy, including } \\
\text { rhabdomyolysis }\end{array}$ & 2 & No & - \\
\hline Carbamazepine & $\begin{array}{l}\text { Dasabuvir, } \\
\text { Ombitasvir, } \\
\text { Paritaprevir } \\
\text { Ritonavir }\end{array}$ & $\begin{array}{l}\text { Decreased dasabuvir, ombitasvir, } \\
\text { paritaprevir and ritonavir exposure; } \\
\text { increased carbamazepine exposure, } \\
\text { induced by ritonavir }\end{array}$ & 2 & $\begin{array}{l}\text { Yes }(1) \\
\text { Caused/Prolonged } \\
\text { Hospitalization (1) }\end{array}$ & - \\
\hline Ethinyl estradiol & $\begin{array}{l}\text { Dasabuvir, } \\
\text { Ombitasvir, } \\
\text { Paritaprevir } \\
\text { Ritonavir }\end{array}$ & Increased risk of ALT elevations & 1 & No & - \\
\hline Flecainide & Ritonavir & Increased risk of cardiac arrhythmias & 1 & $\begin{array}{l}\text { Yes }(1) \\
\text { Life threatening (1), } \\
\text { Caused/Prolonged } \\
\text { Hospitalization (1), } \\
\text { Other (1) }\end{array}$ & $\begin{array}{l}\text { Rhythm } \\
\text { idioventricular (1) }\end{array}$ \\
\hline Ivabradine & Ritonavir & $\begin{array}{l}\text { Increased exposure of ivabradine and } \\
\text { increased risk of QT prolongation. }\end{array}$ & 2 & $\begin{array}{l}\text { Yes }(2) \\
\text { Caused/Prolonged } \\
\text { Hospitalization }(2)\end{array}$ & - \\
\hline Phenobarbital & $\begin{array}{l}\text { Dasabuvir, } \\
\text { Ombitasvir, } \\
\text { Paritaprevir } \\
\text { Ritonavir }\end{array}$ & $\begin{array}{l}\text { Decreased dasabuvir, ombitasvir, } \\
\text { paritaprevir and ritonavir exposure; } \\
\text { increased phenobarbital exposure, induced } \\
\text { by ritonavir }\end{array}$ & 1 & No & - \\
\hline Propafenone & Ritonavir & $\begin{array}{l}\text { Increased risk of arrhythmias or other } \\
\text { serious adverse reactions (CNS depression, } \\
\text { vomiting) }\end{array}$ & 7 & $\begin{array}{l}\text { Yes }(1) \\
\text { Caused/Prolonged } \\
\text { Hospitalization (1), } \\
\text { Other (1) }\end{array}$ & $\begin{array}{l}\text { Atrial fibrillation (1), } \\
\text { Somnolence (1), } \\
\text { Vomiting (1) }\end{array}$ \\
\hline Tacrolimus & Ritonavir & $\begin{array}{l}\text { Increased tacrolimus concentrations and } \\
\text { increased risk of QT-interval prolongation }\end{array}$ & 3 & $\begin{array}{l}\text { Yes }(3) \\
\text { Caused/Prolonged } \\
\text { Hospitalization ( } 3), \\
\text { Life threatening (1), } \\
\text { Other (1) }\end{array}$ & - \\
\hline
\end{tabular}

$\mathrm{N}$ : number of individual case safety reports; ALT = alanine transaminase; CNS = central nervous system.

differences in the exposure and response to drug treatment of women and men, leading to variability in treatment outcomes, because of physiological differences, but also in terms of differences in pharmacokinetics and pharmacodynamics of the medicines [20]. However, the difference could be by chance, as the data analyzed come from a variety of sources. Therefore, these gender differences could be further studied in welldesigned prospective studies, understood and considered in clinical treatment, in order to optimize the patients' treatment outcome.

Even if the elderly patients are defined in most studies as those aged 65 years or older, we considered 70 years old the limit age for the two age groups. Six randomized, multicenter, clinical trials conducted before approval which evaluated the efficacy and safety of $\mathrm{Om} / \mathrm{Pa} / \mathrm{Ri}+\mathrm{Da}$ included patients aged $<70$ years old [21]. Moreover, a previous study conducted on 1,008 Romanian patients with HCV genotype $1 \mathrm{~b}$ compensated cirrhosis assessed the safety of $\mathrm{Om} / \mathrm{Pa} / \mathrm{Ri}+\mathrm{Da}$ in patients aged 70 years and older, compared with patients aged $<70$ years old [15]. Trifan et al. [17] found a higher frequency of AEs reported for patients $\geq 70$ years old ( $37.6 \%$ vs $34.6 \%$ ). Also, more severe ADRs were reported in patients $\geq 70$ years (3.4\%), compared to those $<70$ years $(2.6 \%)$. Death occurred in one patient $(0.9 \%)$ aged 79 years (not related to DAAs therapy), and 6 deaths $(0.7 \%)$ were reported in those under 70 years [15]. Trifan et al. [17] assessed the severity of ADRs; however, in our study data set, the seriousness of ADRs was available, and not the severity. We observed a higher frequency of serious ICSRs and of deaths reported in patients aged $\geq 70$ years old, than in patients aged $<70$ years old.

The importance of identified and potential risks for the products containing $\mathrm{Om} / \mathrm{Pa} / \mathrm{Ri}+\mathrm{Da}$ have been highlighted over time, such as hepatotoxicity (liver damage) and interactions with medicines that have the potential to influence the effectiveness or safety or that may cause serious ADRs [22, 23].

Two Romanian studies reported $40(1.9 \%)$ and four $(0.5 \%)$ cases of hepatic failure, leading to a death rate varying from 35 to $75 \%[13,14]$. Our results showed that for the ICSRs coded with death and/or with life threatening criteria, hepatic failure was the most frequent reported PT and that $44.4 \%$ of the ICSRs coded with hepatic failure led to death. Due to the limitations of our database, we could not establish if the ADRs reported were more likely to be related to the presence of co-morbidities than to the use of $\mathrm{Om} / \mathrm{Pa} / \mathrm{Ri}+\mathrm{Da}$. Cases of hepatic cancer were 
also reported in our VigiBase dataset; however, data on the risk of HCC recurrence or de novo HCC is still limited and needs further investigation $[22,23]$. A study showed that patients treated with $\mathrm{Om} / \mathrm{Pa} / \mathrm{Ri}+\mathrm{Da}$ had the lowest annual HCC incidence $(0.95 \%)$ among four DAAs regimens. However, there were no significant differences between the regimens in HCC risk after treatment [24]. There is still no compelling evidence at present to sustain the conclusion that the use of DAAs for the treatment of $\mathrm{HCV}$ is associated with an increased risk of HCC occurrence $[25,26]$.

Labelled DDIs of co-administered drugs were found to be the most common preventable criterion of ADRs related to DAAs [27]. It is known that especially the medicines that alter the activity of the enzymes CYP3A4 or CYP2C8 are contraindicated in patients treated with $\mathrm{Om} / \mathrm{Pa} / \mathrm{Ri}+\mathrm{Da}$ [16]. Two-thirds of patients with chronic HCV infection were known to be taking at least one medication with the potential for DDIs and DDIs were expected in $66.3 \%$ of patients treated with $\mathrm{Om} / \mathrm{Pa} / \mathrm{Ri}+\mathrm{Da}$ regimen; $8.4 \%$ of patients were taking at least one contraindicated concomitant medicine [28]. In our study, a total of $1.9 \%$ of all ICSRs included nine contraindicated medicines, the majority involving potential alteration of hepatic enzymes. Out of these ICSRs, 38\% were serious. DDIs are a particularly prominent issue in cases of treatment with ritonavir-boosted paritaprevir. Ritonavir is a strong inhibitor of CYP3A4, which boosts the exposure to the protease inhibitor, paritaprevir. However, association of ritonavir with other medicines substrate for CYP3A4 (such as atorvastatin) is contraindicated, because it increases the plasma exposure of the specific concomitant drug, which could lead to serious ADRs. On the other hand, medicines such as carbamazepine, a strong inducer of CYP3A4, could compromise antiviral efficacy [6]. Hepatotoxicity is another important identified risk of Om/ $\mathrm{Pa} / \mathrm{Ri}+\mathrm{Da}$, especially in patient associating medicines that contain ethinylestradiol, which increases the risk of alanine aminotransferase elevation $[22,23]$.

Previous studies outlined the clinical significance of DDIs involving DAAs and the medications mostly used by the HCVinfected patients in clinical practice, which we also found mainly to be potentially interacting for the major DDIs: amlodipine, followed by furosemide, metformin, omeprazole and digoxin $[7,29]$. The results showed that dose adjustments and clinical monitoring should be considered for some medicines: the amlodipine dose should be reduced by half when administered with $\mathrm{Om} / \mathrm{Pa} / \mathrm{Ri}+\mathrm{Da}$; no dose adjustments needed for furosemide, although clinical monitoring is needed; metformin can be associated without dose adjustment; adjusted doses of omeprazole are not required, although may be considered when clinically needed; therapeutic drug monitoring is necessary after adjusting the digoxin dose $[7,25,30]$.

Strict attention to DDIs and their knowledge must be taken into account by healthcare professionals when planning therapy with DAAs in patients with hepatitis C. Discontinuation of contraindicated medications or adjusting doses is sometimes required to assure an effective and safe treatment $[26,31,32]$.

The main strength of the present study was the high number of Romanian safety reports included in the analysis. Efforts are being continuously made to increase the rate of reporting in Romania. In 2008, only 363 spontaneous case reports originating from all sources were reported to the Romanian Agency of Medicines and Medical Devices. However, the number increased over time, and in 2018 it was 6,172 . National online ADR reporting, available since December 2016 , contributes to an increasing number of ADR reports over time [33].

The present analysis has also the advantage to emphasize the serious ADRs, with a detailed analysis of ADRs according to age and gender, and to highlight the important identified and potential risks related to hepatotoxicity and potential DDIs. On the other side, this analysis faced the limits of post-marketing pharmacovigilance system and the characteristics of information registered and available for analysis in the WHO VigiBase, which did not allow access to more information on the patients' history and the case narrative. Also, the possibility of misclassification of some ADRs may be considered, because these ADRs could represent in fact significant complications of the disease, especially when it comes to hepatic disorders [34]. There is the possibility of data omission for the reports, which could have resulted in an underestimation of polytherapy and less identified potential DDIs in this study. Also, there is the possibility that the listed concomitant drugs might not have necessarily been used at the same time as the $\mathrm{Om} / \mathrm{Pa} / \mathrm{Ri}+\mathrm{Da}$ regimen.

In order to screen the treatment for potential DDIs, a user-friendly platform provided by the University of Liverpool can be consulted by healthcare professionals (www.hepdruginteractions.org) together with a mobile application called "Liverpool HEP iChart" [35].

\section{CONCLUSIONS}

In our study, the cases reported for the $\mathrm{Om} / \mathrm{Pa} / \mathrm{Ri}+\mathrm{Da}$ regimen, with or without ribavirin, included serious ADRs, with more cases reported in women than men. Pruritus, fatigue, dizziness and headache were the most commonly reported ADRs. More serious ICSRs were reported in patients aged $\geq$ 70 years old. Hepatitis C patients often present multiple comorbidities, which imply the use of multiple co-medications. The consideration of potential DDIs when using the four active medicines, included in the $\mathrm{Om} / \mathrm{Pa} / \mathrm{Ri}+\mathrm{Da}$ regimen, and increased attention to the association of any medicine for the risk of potential DDIs, is clinically essential.

\section{Conflicts of interest: None to declare.}

Authors' contributions: I.C., R.S., R.D., A.F. and C.M conceived and designed the study. R.S.and R.D. collected the data.I.C., A.F and C.M analysed the data. I.C. drafted the manuscript. All authors critically revised the manuscript, approved the final version to be published, and agree to be accountable for all aspects of the work.

Acknowledgments: The authors would like to thank the Uppsala Monitoring Centre that gave permission to use the data analyzed and to the National Agency for Medicines and Medical Devices of Romania, especially the Romanian National Pharmacovigilance Centre that contributed with data to the VigiBase. The authors would like to acknowledge the contribution of Miruna Muresan, Holceriu Lisandra and Victor Marcu to this study. 
Supplementary material: To access the supplementary material visit the online version of the J Gastrointestin Liver Dis at http://dx.doi. org/10.15403/jgld-2889.

\section{REFERENCES}

1. Manuc M, Preda CM, Popescu CP, et al. New Epidemiologic Data Regarding Hepatitis C Virus Infection in Romania. J Gastrointestin Liver Dis 2017;26:381-386. doi:10.15403/jgld.2014.1121.264.cvr

2. Polaris Observatory HCV Collaborators. Global prevalence and genotype distribution of hepatitis $\mathrm{C}$ virus infection in 2015: a modelling study. Lancet Gastroenterol Hepatol 2017;2:161-176. doi:10.1016/ S2468-1253(16)30181-9

3. Gheorghe L, Sporea I, Iacob S, et al. Position paper on treatment of hepatitis C in Romania, 2017. Part one. J Gastrointestin Liver Dis 2017;26:171-181. doi:10.15403/jgld.2014.1121.262.rom

4. Holmes JA, Rutledge SM, Chung RT. Direct-acting antiviral treatment for hepatitis C. Lancet 2019;393:1392-1394. doi:10.1016/S01406736(18)32326-2

5. Deeks ED. Ombitasvir/Paritaprevir/Ritonavir Plus Dasabuvir: A Review in Chronic HCV Genotype 1 Infection. Drugs 2015;75:1027-1038. doi:10.1007/s40265-015-0412-z

6. Spengler U. Direct antiviral agents (DAAs) - A new age in the treatment of hepatitis C virus infection. Pharmacol Ther 2018;183:118-126. doi:10.1016/j.pharmthera.2017.10.009

7. Badri PS, Dutta S, Wang H, et al. Drug Interactions with the DirectActing Antiviral Combination of Ombitasvir and Paritaprevir-Ritonavir. Antimicrob Agents Chemother 2015;60:105-114. doi:10.1128/ AAC.01778-15

8. Press Releases. 2018 National Health Insurance House. Accessed 10 March 2020.

9. Lindquist M. VigiBase, the WHO global ICSR database system: Basic facts. Ther Innov Regul Sci 2008;42:409-419. doi:10.1177/009286150804200501

10. Aboka D. VigiBase marches on to 17 million reports. Uppsala Reports 2018;3(78). Available from: https://www.who-umc.org/media/164251/ uppsalareports78web.pdf. Accessed: 10 March 2020.

11. Strandell J, Caster O, Bate A, Norén N, Edwards IR. Reporting patterns indicative of adverse drug interactions: a systematic evaluation in VigiBase. Drug Saf 2011;34:253-266. doi:10.2165/11586990-00000000000000

12. European Medicines Agency (EMA). Guideline on good pharmacovigilance practices (GVP). Annex I - Definitions (Rev 4). 9 October 2017. Available from: https://www.ema.europa.eu/en/ documents/scientific-guideline/guideline-good-pharmacovigilancepractices-annex-i-definitions-rev-4_en.pdf. Accessed 10 March 2020.

13. Micromedex ${ }^{\oplus}$ Healthcare Series [Internet database]. Greenwood Village, Colo: Thomson Healthcare. Updated periodically. Accessed 8 January 2020.

14. Fofiu C, Boeriu A, Coman F, et al. Interferon-Free Regimen: Equally Effective in Treatment Naive and Experienced HCV Patients. Ann Hepatol 2019;18:137-143. doi:10.5604/01.3001.0012.7905

15. Preda CM, Popescu CP, Baicus C, et al. Real-world efficacy and safety of ombitasvir, paritaprevir/ $\mathrm{r}+$ dasabuvir+ribavirin in genotype $1 \mathrm{~b}$ patients with hepatitis C virus cirrhosis. Liver Int 2018;38:602-610. doi:10.1111/ liv. 13550

16. Gheorghe L, Iacob S, Curescu M, et al. Real-Life Use of 3 Direct-Acting Antiviral Regimen in a Large Cohort of Patients with Genotype-1b HCV
Compensated Cirrhosis. J Gastrointestin Liver Dis 2017;26:275-281. doi:10.15403/jgld.2014.1121.263.iac

17. Trifan A, Stanciu C, Gheorghe L, et al. Efficacy and safety of paritaprevir/ ritonavir, ombitasvir, and dasabuvir with ribavirin for the treatment of HCV genotype $1 \mathrm{~b}$ compensated cirrhosis in patients aged 70 years or older. Medicine (Baltimore) 2017;96:e9271. doi:10.1097/ MD.0000000000009271

18. El Kassas M, Elbaz T, Hafez E, Esmat G. Safety of direct antiviral agents in the management of hepatitis C. Expert Opin Drug Saf 2016;15:16431652. doi:10.1080/14740338.2017.1240781

19. Ahmed H, Abushouk AI, Menshawy A, et al. Safety and Efficacy of Ombitasvir/Paritaprevir/Ritonavir and Dasabuvir with or without Ribavirin for Treatment of Hepatitis C Virus Genotype 1: A Systematic Review and Meta-analysis. Clin Drug Investig 2017;37:1009-1023. doi:10.1007/s40261-017-0565-5

20. Parekh A, Fadiran EO, Uhl K, Throckmorton DC. Adverse effects in women: implications for drug development and regulatory policies. Expert Rev Clin Pharmacol 2011;4:453-466. doi:10.1586/ecp.11.29

21. Raedler LA. Viekira Pak (Ombitasvir, Paritaprevir, and Ritonavir Tablets; Dasabuvir Tablets): All-Oral Fixed Combination Approved for Genotype 1 Chronic Hepatitis C Infection. Am Health Drug Benefits 2015;8:142-147.

22. European Medicines Agency (EMA). Summary of the risk management plan (RMP) for Viekirax (ombitasvir/paritaprevir/ritonavir). Available from: https://www.ema.europa.eu/en/documents/rmp-summary/ viekirax-epar-risk-management-plan-summary_en.pdf. Accessed 12 February 2020.

23. European Medicines Agency (EMA). Summary of the risk management plan (RMP) for Exviera dasabuvir). Available from: http://docplayer net/99128827-Summary-of-the-risk-management-plan-rmp-forexviera-dasabuvir.html. Accessed 12 February 2020.

24. Mun EJ, Green P, Berry K, Ioannou GN. No difference between direct-acting antivirals for hepatitis $\mathrm{C}$ in hepatocellular carcinoma risk. Eur J Gastroenterol Hepatol 2019;31:47-52. doi:10.1097/ MEG.0000000000001242

25. Antwi SO, Van Houten HK, Sangaralingham LR, Patel T. Risk of De Novo Hepatocellular Carcinoma Following Use of Direct Acting Antiviral Medications for Treatment of Chronic Hepatitis C. Cancer Prev Res (Phila) 2019;12:891-902. doi:10.1158/1940-6207.CAPR-190162

26. Su F, Ioannou GN. Hepatocellular Carcinoma Risk After Direct-Acting Antiviral Therapy. Clin Liver Dis (Hoboken) 2019;13:6-12. doi:10.1002/ cld.781

27. Sessa M, Bernardi FF, Vitale A, et al. Adverse drug reactions during hepatitis $\mathrm{C}$ treatment with direct-acting antivirals: The role of medication errors, their impact on treatment discontinuation and their preventability. New insights from the Campania Region (Italy) spontaneous reporting system. J Clin Pharm Ther 2018;43:867-876. doi:10.1111/jcpt.12744

28. Simon KA, Flisiak R, Łapiński TW, et al. Effect of comedication on ombitasvir/paritaprevir/ritonavir \pm dasabuvir \pm ribavirin therapy in chronic hepatitis C - a real-world study. Clin Exp Hepatol 2019;5:215223. doi:10.5114/ceh.2019.87634

29. Menon RM, Badri PS, Wang T, et al. Drug-drug interaction profile of the all-oral anti-hepatitis $C$ virus regimen of paritaprevir/ritonavir ombitasvir, and dasabuvir. J Hepatol 2015;63:20-29. doi:10.1016/j. jhep.2015.01.026

30. Polepally AR, King JR, Ding B, et al. Drug-Drug Interactions Between the Anti-Hepatitis C Virus 3D Regimen of Ombitasvir, Paritaprevir/ 
Ritonavir, and Dasabuvir and Eight Commonly Used Medications in Healthy Volunteers. Clin Pharmacokinet 2016;55:1003-1014. doi:10.1007/s40262-016-0373-8

31. Klibanov OM, Gale SE, Santevecchi B. Ombitasvir/paritaprevir/ritonavir and dasabuvir tablets for hepatitis $\mathrm{C}$ virus genotype 1 infection. Ann Pharmacother 2015;49:566-581. doi:10.1177/1060028015570729

32. Steiner S, Raguž-Lučić N, Erceg D. Direct-Acting Antivirals (DAAs) Drug-Drug Interactions (DDIs) in the Treatment of Hepatitis C Virus (HCV). IntechOpen 2017. doi:10.5772/intechopen.70788
33. Annual activity reports. Activity report 2018 National Agency for Medicines and Medical Devices of Romania. Available from: https:// www.anm.ro/en/. Accessed 10 March 2020.

34. McGlynn EA, Adams JL, Kramer J, et al. Assessing the Safety of Direct-Acting Antiviral Agents for Hepatitis C. JAMA Netw Open 2019;2:e194765. doi:10.1001/jamanetworkopen.2019.4765

35. HEP drug interactions. Interaction checker. Liverpool Drug Interactions Group. University of Liverpool. Available from: https://www.hepdruginteractions.org/. Accessed 28 September 2020 